\title{
Innovación curricular en la Ciencia Política y la Ciencia Económica: una propuesta desde la Universidad Nacional Abierta y a Distancia - UNAD ${ }^{1}$
}

\author{
Yamile Rivera Romero ${ }^{2}$ \\ Universidad Internacional Menéndez Pelayo \\ Claudia Marcela Rodríguez Rodríguez ${ }^{3}$ \\ Universidad Complutense
}

Recibido: 20/10/2019 - Aprobado 10/12/2019

DOI: https://doi.org/10.22490/26655489.3372

\section{Resumen}

La ciencia política y la ciencia económica, como ciencias sociales, centran su objeto de estudio en relaciones específicas que se producen en el interior de una comunidad. Esto es: las relaciones hombre-hombre enmarcadas por un contexto de poder o un entramado económico. En específico, se distingue el énfasis dado por la ciencia política a las relaciones que se suscitan en los distintos niveles de poder entre los individuos, las instituciones y los dirigentes (Bobbio et al., 1991; O'Donnell, 2010) y para el caso de la economía, las que se generan en el interior del proceso económico (Cuevas, 1997). Luego, ¿cómo puede darse un rompimiento a la forma convencional de diseño curricular para este tipo de programas? y en complemento, ¿cómo puede una disciplina, como la ciencia política o la economía, propiciar cambios en los constructos derivados de las formas tradicionales de concebir las relaciones en el interior de la sociedad?

Este documento expone, en líneas generales, el proceso de diseño curricular seguido por los programas de Economía

\footnotetext{
1 Este documento es resultado del proceso de investigación realizado para el diseño curricular de los programas académicos de Economía (2015) y Ciencia Política (2017) en la Universidad Nacional Abierta y a Distancia - UNAD. Así mismo, este recoge los principales elementos expuestos, en la modalidad de ponencia institucional en el Primer Foro Mundial de Pensamiento Crítico: las luchas por la igualdad, la justicia social y la democracia en un mundo turbulento; organizado por el Consejo Latinoamericano de Ciencias Sociales -CLACSO-(Buenos Aires, Argentina, noviembre 19 a 23 de 2018).

2 Doctorado en Economía y Gobierno (en curso), Universidad Internacional Menéndez Pelayo, Madrid, España. yamile.rivera@unad.edu.co

3 Doctora en Gobierno y Administración Pública, Universidad Complutense de Madrid, Madrid, España. claudia.rodriguez@unad.edu.co
} 
y Ciencia Política, bajo modalidad virtual, propuesto por la Universidad Nacional Abierta y a Distancia - UNAD, el cual se soportó en la metodología de currículo flexible por núcleos problémicos (UNAD, 2011). A través de esta nueva propuesta curricular se espera avanzar en la institucionalización de estas disciplinas en el ámbito nacional y en el reconocimiento de su rol central en los procesos de transformación de la cultura política y el desarrollo económico al brindar formas alternativas para abordar las problemáticas que afectan a los territorios colombianos.

Palabras clave: ciencia política, desarrollo alternativo, economía, innovación curricular, núcleos problémicos, pensamiento crítico

\section{Introducción}

Las ciencias sociales "no son <libres de valores> [...] privilegian al individuo, hacen suyas las libertades humanas y fundamentales de expresión, conciencia y asociación [...]" (Anderson, 2000, p. 202). De acuerdo con esto, la ciencia política y la ciencia económica, como ciencias sociales, centran su objeto de estudio en relaciones específicas que se producen dentro de una comunidad. Esto es: las relaciones hombrehombre enmarcadas por un contexto de poder o un entramado económico. En específico, se distingue el énfasis dado por la ciencia política a las relaciones que se suscitan en los distintos niveles de poder entre los individuos, las instituciones y los dirigentes (Bobbio, 1991; O'Donnell, 2010) y para el caso de la economía, las que se generan en el interior del proceso económico (Cuevas, 1997). Así, surge el interés por establecer cómo a través de la innovación curricular puede una institución educativa promover que sus estudiantes ( $y$ futuros egresados), en su rol de ciudadanos y al servicio de una comunidad, puedan "aspirar a una libertad positiva [...], es decir una libertad que contribuya no solo al despliegue del interés propio sino a metas colectivas que correspondan a las aspiraciones de una sociedad verdaderamente humana [...]" (Bejarano, 2000a, p. 13). En este marco, la Universidad Nacional Abierta y a Distancia - UNAD ${ }^{4}$, institución referente en la formación bajo metodología virtual en Colombia (38 años), durante los últimos años ha iniciado un proceso de transformación del diseño curricular de los

4 La UNAD fue creada mediante la Ley 52 de julio 7 de 1981 como un establecimiento educativo de carácter público del orden nacional, con la denominación Unidad Universitaria del Sur de Bogotá. Posteriormente, mediante la Ley 396 de 1997, su denominación fue modificada por la empleada en la actualidad: Universidad Nacional Abierta y a Distancia. Información adicional sobre la misión, presencia nacional y oferta académica de la UNAD en https://www.unad.edu.co/. 
programas que componen su oferta académica. Estos programas académicos recogen diferentes disciplinas en los niveles de formación: tecnológico, universitario y posgradual.

En este documento, en particular, se presentan los fundamentos que soportaron el diseño de los programas de Economía ${ }^{5}$ y Ciencia Política ${ }^{6}$ con el fin de evidenciar la vigencia y la pertinencia de la nueva apuesta curricular desarrollada por la UNAD. Así, considerando el reto de establecer la articulación entre las necesidades de la sociedad, la academia, la función comunitaria y el desarrollo regional, el diseño curricular para los programas académicos en referencia se sustentó en la metodología de currículo flexible por núcleos problémicos (UNAD, 2011). En este sentido, siguiendo la metodología indicada, el diseño de los programas de Ciencia Política y Economía recogió cinco etapas:

a. Etapa 1: identificación de las situaciones críticas, entendidas como aquellos hechos que han impedido el logro de un desarrollo humano, social y económico sostenible en los territorios.

b. Etapa 2: clasificación de las situaciones críticas identificadas en cuatro subsistemas: social, económico, político y ambiental/ tecnológico.

c. Etapa 3: formulación de los núcleos problémicos (NP) y su articulación en un núcleo integrador de problema (NIP)

d. Etapa 4: presentación de los fundamentos teóricos de cada programa.

e. Etapa 5: concreción de los NP y NIP en redes académicas y plan de estudios.

De acuerdo con lo anterior, el diseño del programa de Economía (Rivera, 2014) partió de reflexionar en torno al principio de actuación de todo agente económico: el principio de racionalidad económica, el cual se soporta en procesos individuales, no colectivos de maximización, privilegiando "el

5 El diseño curricular del programa de Economía se produjo durante el año 2014. Este programa fue aprobado por el Ministerio de Educación Nacional mediante Resolución 10440 de julio 14 de 2015 e inició oferta en el primer semestre de 2016. Información adicional en https://estudios.unad.edu.co/economia

6 El diseño curricular del programa de Ciencia Política se produjo durante el año 2017. Este programa fue aprobado por el Ministerio de Educación Nacional mediante Resolución 002133 del 6 de marzo de 2019 e inicia oferta en el segundo semestre del mismo año. Información adicional en https://estudios.unad.edu.co/ciencia-politica 
egoísmo, el utilitarismo y la eficiencia" (Bejarano, 2000a, p. 12) en contraposición con "la formación de los valores y la aparición y la evolución de la ética social [...], junto con el funcionamiento de los mercados y otras instituciones (Sen, 2013, p. 355). En este sentido, y de conformidad con la metodología de diseño curricular propuesta, se plantearon tres núcleos problémicos con el fin de lograr, sin generar rompimientos con el corpus teórico de la ciencia económica, una aproximación a un principio de racionalidad económica que permita que el economista no solo realice la interpretación de los "fenómenos ocurridos y presentes, sino que se convierta en actor principal en los procesos de transformación y en la apuesta de modelos alternativos de desarrollo económico, que soportados en el desarrollo humano, permitan que el ser humano y las comunidades ocupen un lugar destacado a través del despliegue de sus potencialidades" (Rivera, 2014, p. 14).

Así, los núcleos problémicos planteados (Rivera, 2014) parten de la concepción misma del proceso económico, superando las posturas tradicionales de crecimiento y desarrollo económico, para incorporar la autogestión de la comunidad en conjunción con los servicios prestados por el ecosistema (NP1: El desarrollo económico al servicio del bienestar social, mediante la autogestión comunitaria con responsabilidad ambiental). Otro elemento en consideración es el retomar otras formas de relación entre los agentes económicos, donde adicional al proceso de maximización, de carácter individual, se tome en cuenta a la comunidad y al territorio como espacios alternativos para la organización de la actividad económica, atendiendo a nuevos modelos de gestión, soportados en la asociatividad y la solidaridad, de los recursos económicos e institucionalización de lo económico (NP2: La economía solidaria como alternativa para el desarrollo regional en un contexto globalizado). La propuesta curricular del programa, de igual forma, aboga por fortalecer la relación entre lo público y privado; esto es entre el agente económico tradicional, parte de una comunidad y el Estado, como garante de los derechos y las libertades individuales (NP3: Modelos alternativos de desarrollo desde lo público y lo privado). En conjunto, lo anterior permite establecer como eje articulador o NIP La esencia solidaria garante del desarrollo humano, económico e institucional sostenible.

Por su parte, el programa de Ciencia Política (Rodríguez, 2017) retoma el desarrollo de la teoría contemporánea de la 
ciencia política con énfasis en el análisis político emergente. Así, se presenta un replanteamiento disciplinar desde las teorías propias del sur, que otorgan una mirada a las sinergias dadas en el desarrollo del Estado-nación y del poder en Latinoamérica, y en particular en Colombia. De esta forma, los saberes del sur se tornan en elementos centrales para la comprensión de las problemáticas globales que enfrenta el mundo en la actualidad y el análisis crítico del modelo económico que impacta en el desarrollo de los territorios. En este sentido, el diseño del programa de Ciencia Política (Rodríguez, 2017) integró tres núcleos problémicos (NP).

En primera instancia se aborda la problemática de repensar el desarrollo y el discurso político, logrando establecer diálogos de saberes que permiten orientar la formación del politólogo(a) hacia una nueva construcción epistemológica de la disciplina desde Latinoamérica, soportada en la teoría de la epistemología del sur (NP1: Teoría política emergente: una epistemología desde el sur). El NP2 (Participación ciudadana y transformación local para una administración pública transparente y garante de derechos) busca trazar como elemento central la participación de la sociedad en el accionar del Estado, dado su rol como actor garante de los derechos fundamentales de los ciudadanos. Y, el NP3 (Nuevo orden global: nuevos actores y problemáticas transnacionales) está orientado a profundizar en el estudio de las relaciones internacionales, tomando como punto de partida la realidad de los países que enfrentan problemas comunes y que conforman el denominado Sur Global. Estos tres núcleos convergen en un eje central o NIP: Modelos alternativos de desarrollo para responder a problemáticas en el entorno global, regional y local.

Puesto en estos términos, se espera que, a través de esta nueva propuesta curricular, dada desde los programas de Economía y Ciencia Política ofertados por la UNAD, se logre continuar con el avance en la institucionalización de estas disciplinas en el ámbito nacional y en el reconocimiento de su rol central en los procesos de transformación de la cultura política y el desarrollo económico al brindar formas alternativas para abordar las problemáticas enfrentadas en los territorios colombianos.

De acuerdo con lo presentado, este documento se compone de cuatro partes. La primera es esta introducción. La segunda 
revela los aspectos metodológicos considerados para el diseño curricular de los programas de Economía y Ciencia Política. La tercera expone los fundamentos teóricos que soportan la propuesta curricular de estos programas académicos. Por último, la cuarta ofrece algunas consideraciones finales.

\section{Aspectos metodológicos}

La Universidad Nacional Abierta y a Distancia (UNAD) ha establecido la metodología de currículo flexible por núcleos problémicos "como su modalidad de organización curricular, entendiendo este como el proceso a través del cual la Universidad da respuesta a las necesidades, demandas y oportunidades de sus contextos de actuación y a los vacíos en el desarrollo de las disciplinas" (UNAD, 2011, p. 98). Así, el diseño curricular de los programas de Economía y Ciencia Política se sustentó en la formulación de núcleos problémicos (NP), entendidos estos como:

"Unidades integradoras que posibilitan pensar en procesos de articulación entre los saberes alrededor de objetos de transformación y sugiere estrategias metodológicas que garantizan la síntesis creativa entre la teoría y la práctica. El núcleo problémico es un problema, una necesidad, una oportunidad o un vacío en el conocimiento que aglutina diferentes disciplinas, permitiendo un abordaje integral, un tipo de mediación pedagógica y una propuesta didáctica específica, con el fin de contribuir con la formación del estudiante $y$, a la vez, generar conocimiento pertinente tanto para los contextos sociales como para las mismas disciplinas". (UNAD, 2011, p. 96)

De acuerdo con lo anterior, la fuente de identificación de un núcleo problémico yace en las demandas y las necesidades presentes en los contextos nacional, regional y local, así como en el ámbito de lo disciplinar y lo profesional (ver ilustración 1); esto sumado a las bases trazadas desde las responsabilidades sustantivas y el logro del principio institucional de desarrollo comunitario-regional. 


\section{Ilustración 1. Elementos del currículo flexible en torno a núcleos problémicos.}

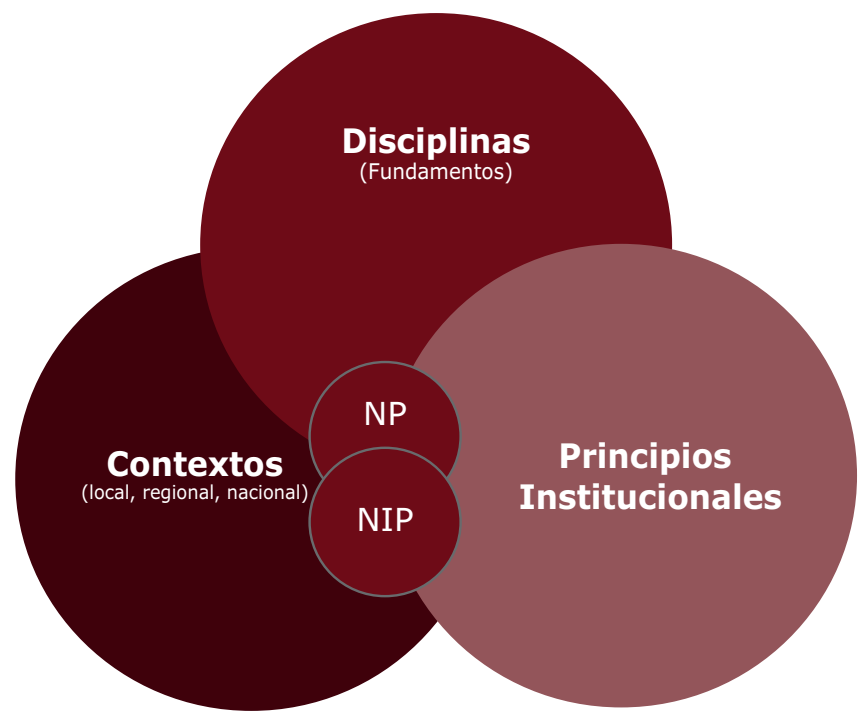

Fuente: UNAD (2011), adaptación propia

En este sentido, el diseño curricular de los programas de Economía (Rivera, 2014) y de Ciencia Política (Rodríguez, 2017) recogió cinco etapas:

Etapa 1: identificación de situaciones críticas de carácter estructural que impactan en el desarrollo social, económico y humano sostenible, con el consecuente impacto sobre la calidad de vida o bienestar de la población en Colombia y Latinoamérica. Para este efecto se realizó un diagnóstico situacional y de actores soportado en la consulta de diversas fuentes y atendiendo a las dimensiones:

a. Contextual, lo cual incluyó la información generada por organismos internacionales, regionales y locales.

b. Epistemológicas (referentes teóricos) propios de cada disciplina (ver ilustración 2). 


\section{Ilustración 2. Análisis contextual y epistemológico.}

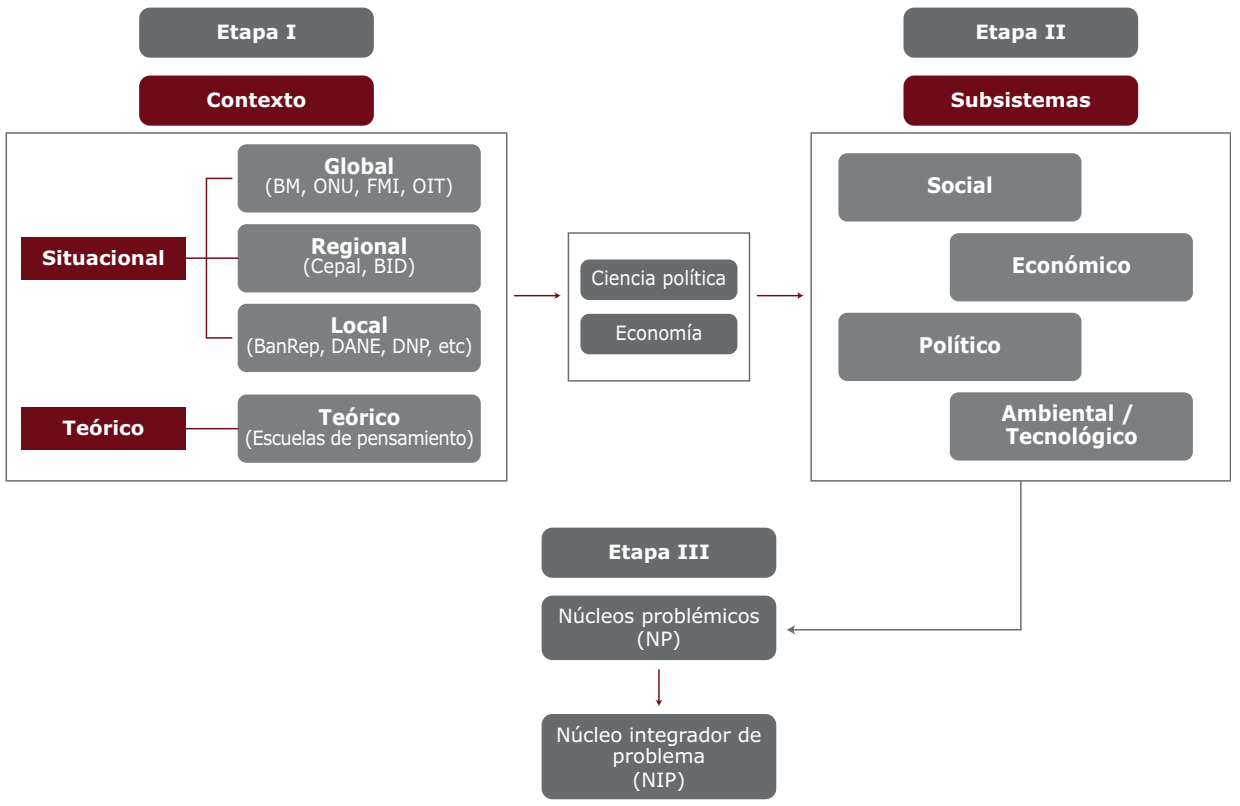

Fuente: Rivera (2014) y Rodríguez (2017), adaptación propia

Esto resulta de gran relevancia, si se considera que:

"Los nuevos horizontes de la formación profesional demandan continuamente la presencia de retos y desafíos para el diseño curricular, el cual debe permitir, sin lugar a duda, la correcta articulación de las dimensiones epistemológicas [y] contextuales, que rigen el currículo, cuya finalidad responda a la formación de un profesional crítico, emergente y proactivo en los campos de actuación". (Mendoza y González, 2015, p. 12)

Etapa 2: clasificación de las situaciones críticas identificadas en cuatro subsistemas: social, económico, político y ambiental/ tecnológico (ver ilustración 3). 


\section{Ilustración 3. Subsistemas de estudio.}

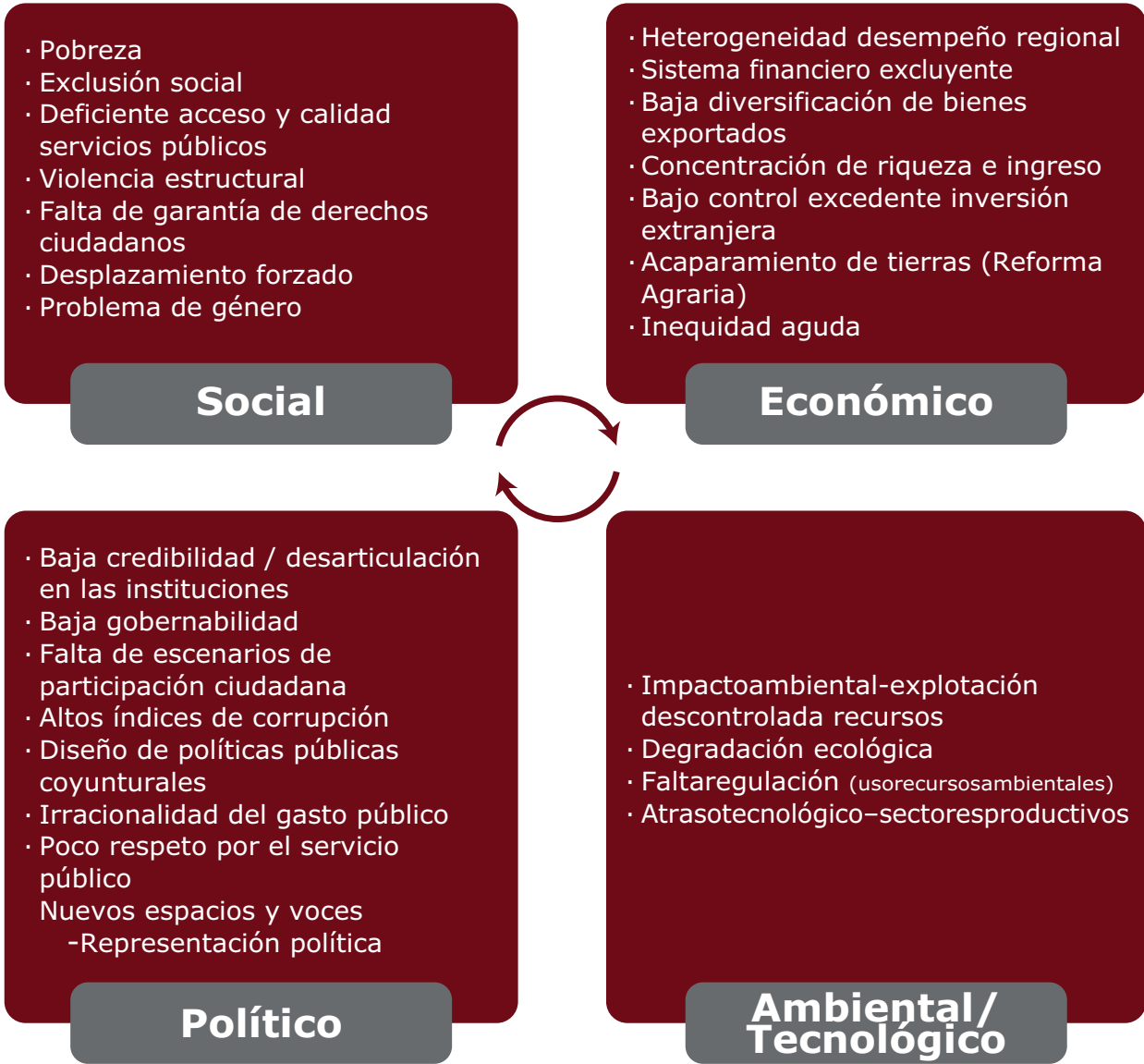

Fuente: Rivera (2014) y Rodríguez (2017), adaptación propia

Etapa 3: formulación de los núcleos problémicos (NP) y su articulación en un núcleo integrador de problema (NIP) siguiendo la participación de diferentes actores.

Etapa 4: presentación de los fundamentos teóricos de cada programa, que serán la base para orientar la formación de los futuros profesionales en economía y ciencia política en la UNAD.

Etapa 5: concreción de los NP y NIP en redes académicas plasmadas en el plan de estudios ${ }^{7}$. En específico, la red académica corresponde al "conglomerado de cursos que permiten el abordaje de un núcleo problémico" (Abadía et al., 2016, p. 8).

7 Debido a que la intencionalidad de este artículo es la presentación del fundamento epistemológico que soporta la propuesta curricular diferenciada de los programas de 


\section{Resultados}

\subsection{Programa de Economía}

El diseño del programa de Economía (Rivera, 2014) partió de la reflexión en torno al principio de actuación de todo agente económico: el principio de racionalidad económica. Este principio "supone que cada persona es capaz de escoger los medios más idóneos para el logro de sus fines, dentro de las restricciones que implica una condición de escasez" (Pérez, 2004, p. 147). Así, el interés está en los procesos individuales asociados con la maximización del bienestar (consumidor) y el beneficio (productor) en el marco de los incentivos de interacción e intercambio provistos por el mercado. Por tanto, este principio está privilegiando "el egoísmo, el utilitarismo y la eficiencia" (Bejarano, 2000a, p. 12) en el interior del proceso económico. Esta tríada de elementos hace parte de las reflexiones llevadas a cabo en el interior de la economía, como ciencia, a partir de las últimas décadas del siglo $\mathrm{XX}$, puesto que resulta de importancia:

"Intentar mejorar, desde un punto de vista ético, los resultados del proceso económico y de la asignación de recursos mejorando el sistema de valores, los ámbitos de solidaridad y el sistema institucional sin pretender interferir en el núcleo invariante de las leyes del mercado, porque, como ha probado la experiencia histórica, esa interferencia puede acabar, en nombre de la justicia social, sacrificando la libertad que es el principal de los bienes del hombre". (Bejarano, 2000a, p. 14)

En este marco se establece que las elecciones tomadas por los agentes económicos no solo responden al movimiento de los precios relativos experimentados en los mercados de bienes o servicios (Ayala, 1999). Estas elecciones se ven influenciadas por las señales y estímulos provenientes de los entornos jurídico, económico, social y político a los que deben sujetarse los individuos. Por tanto, si bien se está en presencia de la autorregulación del mercado, esta debe producirse en consonancia con el logro de la prosperidad material procurando el bien común (Bejarano, 2000a). Puesta en estos términos, la prosperidad económica "puede concebirse [...] como un proceso de expansión de las libertades reales [o fundamentales] de las que disfrutan los individuos" (Sen, 2013, p. 19). No obstante,

Economía y Ciencia Política, no se hará la incorporación de los planes de estudio de estos. Pueden ser consultados en: https://estudios.unad.edu.co/economia/plan-de-estudios y https://estudios.unad.edu.co/ciencia-politica/plan-de-estudios, respectivamente. 
se hace hincapié en que este tipo de libertad, considerada como positiva, debe contribuir "no solo al despliegue del interés propio sino a metas colectivas que correspondan [con] las aspiraciones de una sociedad verdaderamente humana, en la que el hombre encuentre condiciones de autorrealización" (Bejarano, 2000a, p. 13).

De conformidad con lo anterior, la libertad se constituye en un elemento clave para la definición de las bases que soportan el desarrollo socioeconómico sostenido de un territorio, en el entendido que el territorio "es entonces más que una red, es la constitución de un espacio abstracto de cooperación entre diferentes actores con un anclaje geográfico para engendrar recursos particulares y soluciones inéditas" (Pecqueur, 2000, p. 15 en Martínez, 2012, p. 13). Luego se sigue que el territorio se constituye en el escenario en que confluyen las diferentes relaciones sociales a nivel microterritorial (entre individuos) y macroterritorial (con otros territorios). Por tanto, se aduce que el desarrollo depende de la libre agencia o elección de los individuos; esto es la libertad de que dispone todo individuo para ser partícipe activo (persona-sujeto) en los procesos de toma de decisiones de carácter social, actuando como promotor y gestor del desarrollo (Max-Neef, 1998); esto es,

[...] los individuos pueden configurar en realidad su propio destino y ayudarse mutuamente. No tienen por qué concebirse como receptores pasivos de las prestaciones de ingeniosos programas de desarrollo. Existen, de hecho, poderosas razones para reconocer el papel positivo que desempeña la agencia libre y viable, e incluso la impaciencia constructiva. (Sen, 2013, p. 28)

En este orden de ideas, se establece que las estrategias para propiciar, promover y fortalecer el desarrollo deben emerger desde la comunidad (la base), soportada en las capacidades económicas, sociales, culturales y a Ahn, 2003, p. 156). En este escenario se soporta la propuesta del programa de Economía, cuyo planteamiento curricular procuró recoger aquellas formulaciones teórico-prácticas que permiten: "[...] dar cuenta de un conjunto significativo de experiencias económicas [...] que comparten algunos trazos constitutivos y esenciales de solidaridad, mutualismo, cooperación y autogestión comunitaria, [definiendo así] una racionalidad especial, diferente de otras racionalidades económicas" (Razeto, 1993, p. 40 en Lechat, 
2002, p. 130, traducción propia). Por consiguiente, la propuesta curricular propuesta para el programa de Economía revitaliza la premisa: "la economía está para servir a las personas y no las personas a la economía" (Max-Neff, 1998, p. 46), para lo cual busca otorgar a los estudiantes las herramientas teóricoanalíticas que permitan generar las bases para el planteamiento de nuevos modelos alternativos de desarrollo y que propendan por el equilibrio socioeconómico-ambiental en el territorio.

De acuerdo con lo anterior, y en aplicación de la metodología de diseño curricular propuesta (currículo flexible por núcleos problémicos), para el diseño del programa de Economía (Rivera, 2014) se plantearon tres núcleos problémicos (NP) tal como sigue en la ilustración 4:

\section{Ilustración 4. Núcleos problémicos / Núcleo integrador de problema.}

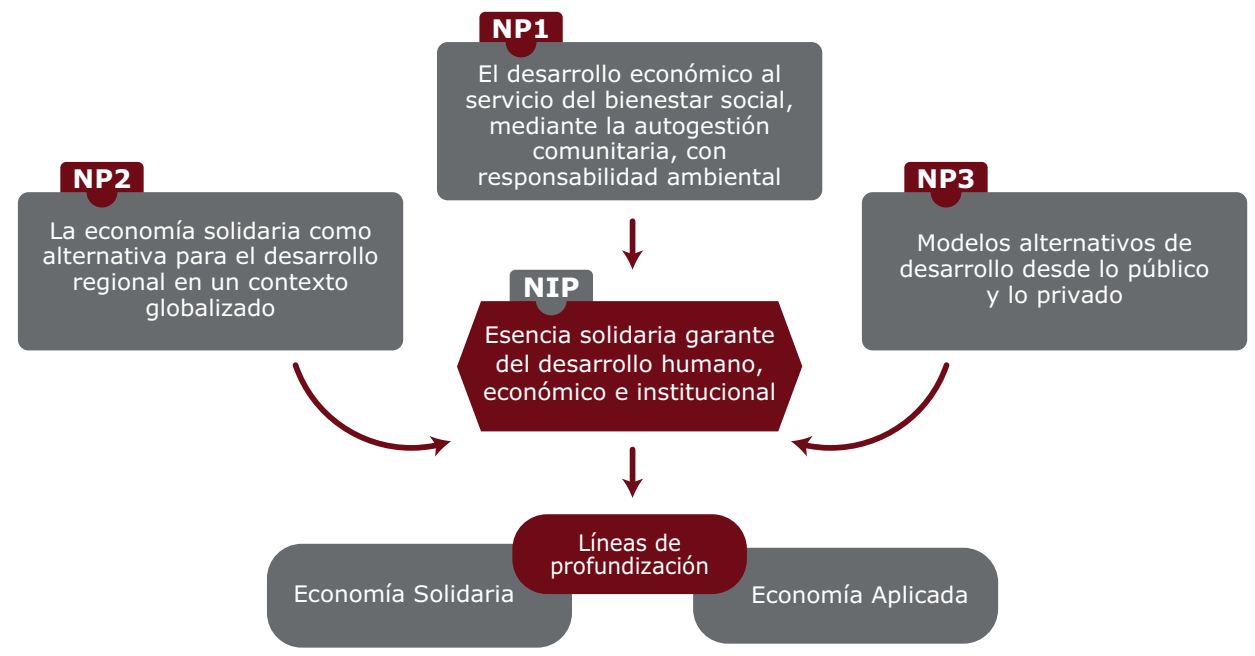

Fuente: Rivera (2014), adaptación propia

NP1: El desarrollo económico al servicio del bienestar social, mediante la autogestión comunitaria, con responsabilidad ambiental. Partiendo de la concepción misma del proceso económico y superando las posturas tradicionales de crecimiento y desarrollo económico, se considera de importancia la incorporación de la autogestión de la comunidad en conjunción con los servicios prestados por el ecosistema para el logro de un desarrollo sostenido puesto que "ningún proceso económico 
puede ocurrir al margen de los servicios que prestan los ecosistemas" (Max-Neef, 2006, 23). Esto conlleva al tratamiento de estos servicios como un bien común inmersos en sistemas socioecológicos, considerados estos últimos como: "aquellos sistemas en los que se presentan interacciones [funcionales y espaciales] entre sistemas sociales y ecológicos" (Salas et al., 2011, p. 137). Ver ilustración 5.

\section{Ilustración 5. Sistemas socioecológicos.}

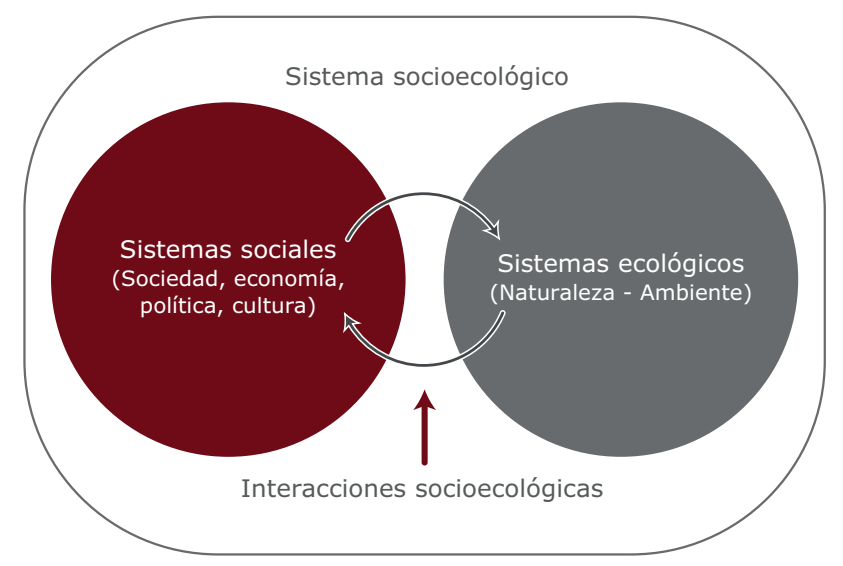

Fuente: Salas (2011, p. 138), adaptación propia

Así, surge la noción de comunidad autogestora como actor dinamizador del desarrollo sostenible. Por tanto, se está en presencia de un desarrollo a escala humana que se centra en la atención y satisfacción de las necesidades fundamentales del ser humano, la autodependencia y la articulación hombrenaturaleza-tecnología (Max-Neef, 1998). En este sentido el programa de Economía a través de su apuesta curricular procura:

"Abordar la problemática del bienestar de la población desde un enfoque empírico y académico, logrando establecer diálogos de saberes que permitan orientar la formación del futuro economista hacia la construcción de nuevos modelos de desarrollo que contribuyan a la modernización de las instituciones, el planteamiento de políticas públicas coherentes y la optimización de la gestión económica". (Rivera, 2014, p. 74) 
NP2: La economía solidaria como alternativa para el desarrollo regional en un contexto globalizado. Otro elemento en consideración es el retomar otras formas de relación entre los agentes económicos, donde adicional al proceso de maximización, de carácter individual, se incorporen a la comunidad y el territorio como espacios alternativos para la organización de la actividad económica, atendiendo a nuevos modelos de gestión, soportados en la asociatividad y la solidaridad, de los recursos económicos e institucionalización de lo económico. Esto se inscribe en la nueva la relación Estado-mercado-sociedad en la cual se ha dado cabida a una forma emergente de organización, denominada economía solidaria, cuyo centro de actuación es la comunidad a través de las relaciones y demandas sociales producidas en su interior, en conjunción con las potencialidades del territorio.

De esta forma se pone de presente el debate si el mercado, como mecanismo regulador de los agentes económicos y el Estado, como ente garante de las libertades y derechos individuales y colectivos, pueden generar procesos inclusión para el número de significativo de ciudadanos carentes de los recursos económicos y las capacidades humanas suficientes para atender sus necesidades básicas individuales y las de su núcleo familiar. Por consiguiente, el programa ha tomado como una de sus líneas de profundización la economía solidaria, "a partir de cuya experiencia se desea procurar la construcción de modelos alternativos de desarrollo basados en las potencialidades del ser humano y las comunidades; así como en la construcción del marco institucional básico para el desarrollo humano sostenible" (Rivera, 2014, p. 76).

NP3: Modelos alternativos de desarrollo desde lo público y lo privado. La propuesta curricular del programa, de igual forma, aboga por fortalecer la relación entre lo público y lo privado; esto es, entre el agente económico tradicional, parte de una comunidad y el Estado. Este hecho resulta válido si se considera que "desde distintas perspectivas y por muy diversas razones, han surgido varios interrogantes y temas de reflexión [...] sobre la pertinencia del conocimiento que generan las comunidades académicas, sobre todo en ciencias sociales, y [...] la transmisión de ese conocimiento" (Bejarano, 2000b, p. 219). Por tanto, "surge la necesidad de formar profesionales competentes que contribuyan de manera efectiva y significativa al fortalecimiento de los cimientos de la teoría y práctica de la Ciencia Económica; 
así como a la transformación del contexto inmediato" (Rivera, 2014 , p. 77) como fundamento para promover el desarrollo humano, económico e institucional sostenible. De esta forma, se generan los elementos base para cerrar las actuales brechas y desigualdades sociales presentes en Colombia.

En conjunto, lo anterior permite establecer como eje articulador La esencia solidaria garante del desarrollo humano, económico e institucional sostenible (núcleo integrador de problema). Esto permite que dentro del programa se establezca un diálogo entre el discurso científico, propio de la economía y el discurso ético, característico de la solidaridad (Razeto, 1999).

\subsection{Programa de Ciencia Política}

El programa de Ciencia Política (Rodríguez, 2017) retoma el desarrollo de la teoría contemporánea de la ciencia política, con énfasis en el análisis político emergente. Así, se presenta un replanteamiento disciplinar desde las teorías propias del sur, que otorgan una mirada a las sinergias dadas en el desarrollo del Estado-nación y del poder en Latinoamérica, y en particular en Colombia. De esta forma, los saberes del sur se tornan en elementos centrales para la comprensión de las problemáticas globales que enfrenta el mundo en la actualidad y el análisis crítico del modelo económico que impacta en el desarrollo de los territorios.

De lo anterior se establece que la disciplina de la ciencia política, tanto en el ámbito nacional como internacional, requiere de una revisión profunda, partiendo de sus principios, postulados y normatividades, en el contexto de la descolonización de los Estados y de todos sus procesos, los cuales aún conservan trazos coloniales. Si bien, según Quijano (2014), las administraciones coloniales vieron su fin con las guerras de independencia, las estructuras de poder de los viejos imperios coloniales se mantienen intactas en la actualidad, en lo que se ha denominado "la colonialidad del poder" 0 , expresado en otros términos, el mantenimiento de las estructuras coloniales a través del tiempo en los Estados y sus instituciones (como la familia, la escuela y la economía) y, por supuesto, en el ejercicio de la política y la regulación mediante el derecho de las dinámicas políticas. 
En este sentido, es prioritario el repensar el reforzamiento del Estado-nación colombiano desde la propuesta de una:

"Política de la liberación como una solución desde la alteridad latinoamericana a los problemas que suscitan las malas administraciones públicas de los Estados nación como Colombia, es indispensable conocer cómo una propuesta centrada en la ética; funciona para desarrollar un nuevo tipo de gobierno, más democrático, más participativo y ante todo menos excluyente. Es la posibilidad de salir del círculo vicioso que crearon las administraciones coloniales para la explotación de los pueblos latinoamericanos y considerar una nueva etapa de desarrollo desde la correcta administración de los recursos para la prosperidad". (Barragán, 2011, p. 109)

En tal contexto, si se acepta que "Desde la educación comunitaria [estrategia fundamental en el actuar de la UNAD] hay que reconocer al 'otro' y a los 'otros' dentro de la convivencia pacífica, para construir el cambio en las interrelaciones sociales y concebir el conflicto social como una oportunidad o mediación pedagógica" (UNAD, 2011, p. 162), es innegable que la educación política que imparta la UNAD ha de poseer una matriz teórica distinta a la que fundamenta a la mayoría de escuelas de ciencia política en el país. Por tanto, la aceptación de una perspectiva diferenciada, como la que propone el programa de Ciencia Política de la UNAD, soportada en el planteamiento de espacios y prácticas que reestructuren las dinámicas de poder propias de los contextos académicos de la disciplina y del contexto inmediato del estudiante-egresado, contribuyen a impulsar nuevas formas de actuación en los ámbitos jurídico-políticos, posibilitando así que los ciudadanos ejerzan sus derechos y deberes y por esta vía puedan acceder a los procesos de toma de decisión en la esfera política del país. En cumplimiento de esto, el programa de Ciencia Política pretende brindar respuesta a las problemáticas de los territorios, generando profesionales que converjan desde el discurso de desarrollo alternativo y las epistemologías del sur, recogiendo lo local como parte fundamental para la transformación y el desarrollo de las comunidades, del entorno y de las problemáticas trasnacionales, con un fuerte valor ético y garante de derechos. 
De acuerdo con lo anterior, y en aplicación de la metodología de diseño curricular propuesta (currículo flexible por núcleos problémicos), para el diseño del programa de Ciencia Política (Rodríguez, 2017) se plantearon tres núcleos problémicos (NP) tal como sigue en la ilustración 6:

\section{Ilustración 6. Núcleos problémicos / Núcleo integrador de problema.}

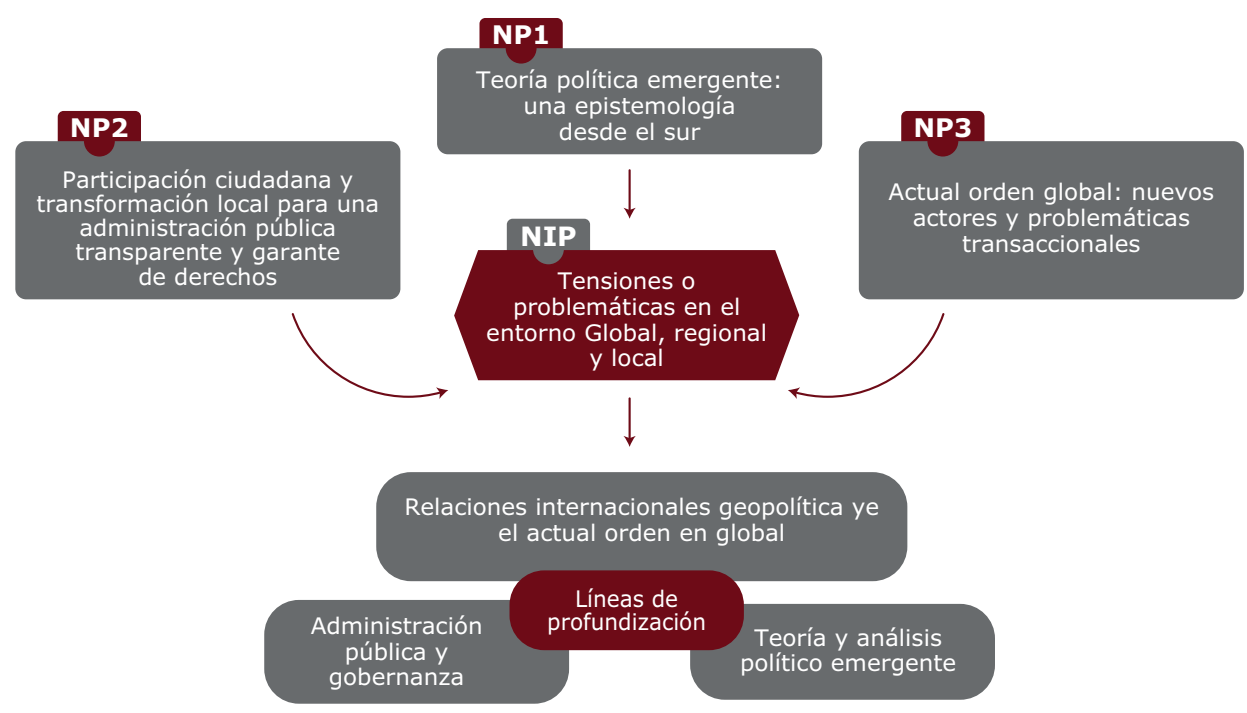

Fuente: Rodríguez (2017), elaboración propia

NP1: Teoría política emergente: una epistemología desde el sur. En primera instancia, se aborda la problemática de repensar el desarrollo y el discurso político, logrando establecer diálogos de saberes que permiten orientar la formación del politólogo hacia una nueva construcción epistemológica de la disciplina desde Latinoamérica, soportada en la teoría de la epistemología del sur.

Esta otra epistemología que tiene su génesis en la Teoría Crítica y se recrea en América Latina, desde el Sur, se asume desde la praxis de un logo emancipador que fractura los límites hegemónicos del "capitalismo sin fin" y del "colonialismo sin fin", ya que hace posible recuperar desde la "sociología de las emergencias", la presencia de los pueblos milenarios que han logrado la recreación de su hábitat a través de una relación simbiótica directa, con los 
ciclos o procesos de génesis y muerte de la Madre Tierra (Pachamama). (De Sousa, 2011, p. 17).

Así expresado, este núcleo problémico pretende responder a la pregunta de si es posible desde el análisis teórico-político dar respuesta a procesos alternativos de desarrollo político, económico y social. Por ello, se tiene como objetivo poner en el centro del análisis todos aquellos campos del conocimiento que se sitúan en la periferia del paradigma epistémico eurocéntrico, en gran medida. Esto es, el análisis desde las coordenadas de los saberes damnificados por la hegemonía del pensamiento único colonial, clasista, patriarcal y racista.

NP2: Participación ciudadana y transformación local para una administración pública transparente y garante de derechos. Se busca trazar como elemento central la participación de la sociedad en el accionar del Estado, dado su rol como actor garante de los derechos fundamentales de los ciudadanos. Esto es relevante si se considera que la participación ciudadana en el control de la gestión pública es uno de los componentes de modernización y fortalecimiento de la capacidad institucional del sector público, siempre que se encuentren soportados en la Constitución Política los desarrollos legislativos y las políticas del Estado (Procuraduría General de la Nación et al., 2011). En ese marco:

"La participación lleva a incorporar en la esfera pública nuevos problemas y nuevas soluciones, asociados a identidades (étnicas, de género, de clase social) y con perspectivas de institucionalizar la inclusión social para que se garanticen derechos. [...] La participación protagónica de la población puede revertir estos procesos sociohistóricos en la configuración del Estado, además de promover un cambio institucional sostenido en el tiempo". (Ortiz, 2012, p. 2)

NP3: Nuevo orden global - nuevos actores y problemáticas transnacionales. Una de las dinámicas claves que está en la base de las transformaciones que ha experimentado la sociedad internacional es la creciente interdependencia que se ha generado en las relaciones internacionales a todos los niveles y en todos los ámbitos (Del Arenal, 2012). Al respecto: 
[Una] "expresión clara de la interdependencia es el carácter común y global de muchos de los problemas de la sociedad mundial, frente a los cuales las políticas nacionales o individuales de los actores nada pueden hacer, siendo necesarias políticas comunes y globales, basadas en la coordinación de políticas nacionales". (Arenal, 2012, p. 33)

En ese marco, el programa de Ciencia Política se ha propuesto profundizar en el estudio de las relaciones internacionales tomando como punto de partida la realidad de los países que enfrentan problemas comunes y que conforman el denominado Sur Global.

Estos tres núcleos convergen en un eje central (o NIP): "Se identifican tensiones o problemáticas en el entorno global, regional y local (ambientales, nuevos escenarios de actuación, actores, sistemas de representación política) que requieren modelos alternativos de desarrollo que respondan a las sinergias locales, nacionales, internacionales (trasnacionales), supranacionales" (Rodríguez, 2017, p. 103).

\section{Consideraciones finales}

El proceso de innovación curricular "por núcleos problémicos" promovido por la UNAD se convirtió en un escenario de análisis de los fundamentos teóricos en los que se soportan la economía y la ciencia política en conjunción con la observación sistémica de la dinámica social, económica, cultural y política registrada en los territorios colombianos y las perspectivas epistemológicas y de la praxis en estas disciplinas. Así, la información obtenida de estos ámbitos (como disciplinar, contextual y del ejercicio profesional) dio origen a un conjunto de núcleos estructurantes de una propuesta curricular alternativa para la formación de profesionales en las áreas de economía y ciencia política cuyo propósito no solo se centra en interpretar los hechos económicos y políticos acaecidos y presentes en Colombia "sino [en] la intencionalidad de proponer modelos alternativos de desarrollo [socio-económico...], donde el ser humano y las comunidades ocupen un lugar destacado a través del despliegue de sus potencialidades" (Rivera, 2014, p. 14) en total armonía con los sistemas ecológicos. Por tanto, con la propuesta curricular presentada se espera avanzar en la institucionalización de estas 
disciplinas en el ámbito nacional y en el reconocimiento de su rol central en los procesos de transformación de la cultura política y el desarrollo económico al brindar formas alternativas para abordar las problemáticas que afectan la realidad nacional.

\section{Referencias}

Abadía, C., Vela, P. y Montero, R. (2016). Lineamientos Generales del Currículo en la UNAD. Serie Lineamientos Microcurriculares en la UNAD V2. Bogotá: Universidad Nacional Abierta y a Distancia - UNAD, p. 39. Recuperado de https://viaci. unad.edu.co/images/Docsoficiales/Lineamientosmicro.pdf

Anderson, L. (2000). Las ciencias sociales bajo presión. Revista de Economía Institucional, 2 (2), primer semestre, pp. 197-203. Recuperado de http://www.redalyc. org/articulo.oa?id $=41900212$

Ayala, J. (1999). Instituciones y economía. Una introducción al Neoinstitucionalismo económico. México D.F.: Fondo de Cultura Económica, p. 397.

Barragán, D. (2012). La política de la liberación. Revista Vía Luris, (12), pp. 105-117. Recuperado de https://revistas.libertadores.edu.co/index.php/ViaIuris/article/view/100

Bejarano, J. (2000a). Ética y economía. Revista de Economía del Rosario, 3 (1), pp. 11-14. Recuperado de https://revistas.urosario.edu.co/index.php/economia/ article/view/4229/3092

Bejarano, J. (2000b). Guía para la evaluación del estado de las ciencias sociales en CoIombia. Revista Economía Institucional, 2 (3), segundo semestre, pp. 219-228. Recuperado de https://www.economiainstitucional.com/pdf/No3/jbejarano3.pdf

Bobbio, N., Matteucci A., y Pasquino, G. (1991). Diccionario de Política. México: Siglo XXI Ed., pp. 255-263.

Congreso de la República de Colombia. (1981). Ley 52 de julio 7 de 1981. Bogotá: Ministerio de Educación Nacional / Ministro de Hacienda y Crédito Público. Recuperado de http://www.suin-juriscol.gov.co/viewDocument.asp?id=1606318

Congreso de la República de Colombia. (1997). Ley 396 de agosto 5 de 1997. Bogotá: Ministerio de Educación Nacional / Ministro de Hacienda y Crédito Público. Recuperado de http://www.secretariasenado.gov.co/senado/basedoc/ley_0396_1997.html

Cuevas, H. (1997). Introducción a la Economía. Bogotá: Universidād Externado de Colombia, p. 765.

De Soussa, B. (julio-septiembre de 2011). Epistemologías del Sur. Utopía y Praxis Latinoamericana, 16 (54), pp. 17-39. Recuperado de http://www.redalyc.org/ pdf/279/27920007003.pdf

Lechat, N. (junio de 2002). Economia social, economia solidária, terceiro setor: do que se trata? Civitas - Revista de Ciências Sociais, 2 (1), pp. 23-140. Recuperado de http://www.redalyc.org/pdf/742/74220108.pdf

Martínez, L. (enero-abril de 2012). Apuntes para pensar el territorio desde una dimensión social. Ciências Sociais Unisinos, 48 (1), pp. 12-18. Recuperado de http:// www.redalyc.org/pdf/938/93823702003.pdf

Max-Neef, M. (1998). Desarrollo a escala humana. Conceptos, aplicaciones y algunas reflexiones. Barcelona: Icaria Editorial S.A., p. 148. Recuperado de http://www. max-neef.cl/descargas/Max_Neef-Desarrollo_a_escala_humana.pdf

Max-Neef, M. (2006). El poder de la globalización. Revista Facultad Nacional de Salud Pública, (24), número especial, pp. 23-29. Recuperado de https://aprendeenlinea. udea.edu.co/revistas/index.php/fnsp/article/view/26459/20779610

Mendoza, F. y González, T. (2015). Fuentes epistemológicas y contextuales para la generación de currículos pertinentes en la sociedad del conocimiento. Foro Educacional 24, pp. 11-33. Recuperado de https://dialnet.unirioja.es/servlet/articulo?codigo $=6429423$

Ministerio de Educación Nacional. (2015). Resolución 10440 de julio 14 de 2015. Bogotá: Universidad Nacional Abierta y a Distancia. Recuperado de https://estudios.unad. edu.co/images/ecacen/Econom\%C3\%ADa/Registro_Calificado_Economia.pdf 
Ministerio de Educación Nacional. (2019). Resolución 002133 de marzo 6 de 2019. Bogotá: Universidad Nacional Abierta y a Distancia. Recuperado de https://estudios. unad.edu.co/images/ecjp/CP-Resolucion-002133-del-6-de-marzo-de-2019.pdf

O'Donnell, G. (2010). Democracia, agencia y Estado. Teoría con intención comparativa. Buenos Aires: Editorial Prometeo, p. 375.

Ortiz, L. (2012). La instancia pública de la gestión. Algunas consideraciones sobre la participación ciudadana en el Estado. Revista Reforma y Democracia - Centro Latinoamericano de Administración para el Desarrollo, (54), pp. 179-194. Recuperado de https://www.redalyc.org/pdf/3575/357533686007.pdf

Ostrom, E. y Ahn, T. (enero-marzo de 2003). Una perspectiva del capital social desde las ciencias sociales: capital social y acción colectiva. Revista Mexicana de Sociología, 65 (1), pp. 155-233. Recuperado de http://www.scielo.org.mx/pdf/rms/ v65n1/v65n1a5.pdf

Pérez, M. (2004). La economía en el panorama de las ciencias sociales. Variaciones sobre un tema de Bejarano. Cuadernos de Economía, 28 (40), pp. 143-173. Recuperado de http://www.scielo.org.co/pdf/ceco/v23n40/v23n40a07.pdf

Procuraduría General de la Nación, Defensoría del Pueblo, Superintendencia de Industria y Comercio y Confederación Colombiana de Consumidores. (2011). Memorias del programa de capacitación en participación ciudadana y control social: En la garantía de derechos y en la contratación estatal. Bogotá: Pro-offset Editorial S.A., p. 126. Recuperado de https://www.procuraduria.gov.co/portal/media/ file/memorias\%20del\%20programa.pdf

Quijano, A. (2014). Colonialidad del poder, eurocentrismo y América Latina. En: Cuestiones y horizontes: de la dependencia histórico-estructural a la colonialidad/ descolonialidad del poder. Buenos Aires: Consejo Latinoamericano de Ciencias Sociales - CLACSO, pp. 777-832. Recuperado de http://biblioteca.clacso.edu. ar/clacso/se/20140507042402/eje3-8.pdf

Razeto, L. (1999). La economía de la solidaridad: Concepto, realidad y proyecto. Revista Persona y Sociedad, 13 (2), p. 17. Recuperado de http://www.luisrazeto.net/ content/la-econom\%C3\%ADa-solidaria-concepto-realidad-y-proyecto

Rivera, Y. (2014). Documento maestro de condiciones de calidad con fines de obtención del registro calificado para el programa de Economía. Bogotá: Universidad Nacional Abierta y a Distancia, p. 377.

Rodríguez, C. (2017). Documento maestro de condiciones de calidad con fines de obtención del registro calificado para el programa de Ciencia Política. Bogotá: Universidad Nacional Abierta y a Distancia, p. 262.

Salas, W., Ríos, L. y Álvarez, J. (2011). Bases conceptuales para una clasificación de los sistemas socioecológicos de la investigación en sostenibilidad. Revista Lasallista de Investigación, 8 (2), pp. 136-142. Recuperado de http://www.redalyc.org/ comocitar.oa?id $=69522607015$

Sen, A. (2013). Desarrollo y libertad. Bogotá: Editorial Planeta Colombiana S.A., p. 440.

Stöhr, W. (4 de junio de 1978). Development from below: the bottom-up and periphery-inward development paradigm. IIR-Discussion Papers, pp. 1-55. Recuperado de http://epub.wu.ac.at/6180/1/IIR_Disc_04.PDF

Universidad Nacional Abierta y a Distancia. (2011). Proyecto Académico Pedagógico Solidario - PAPS- Versión 3.0. Bogotá: Ediciones Hispanoamericanas Ltda., p. 197. 